\title{
Emerging sensor-based m-health interventions in the assessment of psychotic symptoms
}

\section{Bulgheroni, Ab.Acus srl}

This speech aims to overview ongoing research trends on the integration of mobile health and sensors based behavioral analysis in therapeutics programs for subjects with mental health symptoms or disorders. The variety of easily acquirable personal data by smartphones and wearables in a transparent and unobtrusive way, offers the opportunity to describe the person in terms of his/her lifestyle and behavior at physical, cognitive and environmental level. An appropriate management of these data may initiate a new line in healthcare management characterized by tailored and timely interventions. However, despite the huge amount of data that could be acquired, an effective contribution of such information to the improvement of the quality of care in mental-health is still not sufficiently explored. The sensors and data which have been used in studies on mental status include accelerometer, gyroscope, GPS, microphone, calls, messages, screen, apps usage, environmental light, heart rate, skin conductance, and temperature. The primary outcomes build on correlations between sensor data and mental health status/severity of symptoms. These data are provided from studies on bipolar disorders and depression, using validated clinical scales (Patient Health Questionnaire-9; Hamilton Rating Scale for Depression; Young Mania Rating Scale; Center for Epidemiologic Studies Depression Scale; etc.).

m-RESIST consortium is fully aware of the importance to describe behavioral patterns of patients with schizophrenia that could be used to setup remotely based therapeutic tool. m-RESIST is setting up a framework for the creation of a Clinical Decision Support System based on a mobile therapeutic intervention for treatment-resistant schizophrenia 